\title{
Prenatal exposure to perfluoroalkyl substances and thyroid hormone concentrations in cord plasma in a Chinese birth cohort
}

\author{
Hong Liang', Ziliang Wang ${ }^{1}$, Maohua Miao', Youping Tian², Yan Zhou³, Sheng Wen³, Yao Chen',
}

Xiaowei Sun ${ }^{1}$ and Wei Yuan ${ }^{1 *}$ (D)

\begin{abstract}
Background: Evidence of associations between prenatal exposure to perfluoroalkyl substances (PFASs) and fetal thyroid hormones (THs) is controversial, and few studies have estimated the associations, while addressing the high correlations among multiple PFASs. We aimed to examine the associations between prenatal PFAS exposure and thyroid hormone concentrations in cord blood.

Methods: A total of 300 mother-infant pairs from the Shanghai-Minhang Birth Cohort Study were included. We measured the concentrations of eight PFASs in maternal plasma samples collected at 12-16 gestational weeks, as well as those of total thyroxine (T4), free T4 (FT4), total triiodothyronine (T3), free T3 (FT3), and thyroid stimulating hormone (TSH) in cord plasma. We estimated the associations between maternal PFAS concentrations and TH concentrations using linear regression and Bayesian kernel machine regression (BKMR) models.
\end{abstract}

Results: In BKMR models, higher PFAS mixture concentrations were associated with increased T3 concentrations, and there were suggestive associations with increased FT3 concentrations. For single-exposure effects in BKMR models, a change in PFDA, PFUdA, and PFOA concentrations from the 25th to 75th percentile was associated with a 0.04 (95\%Crl: - 0.01, 0.09), $0.02(95 \% \mathrm{Crl}:-0.03,0.07)$, and 0.03 (95\% Crl: - 0.001, 0.06) nmol/L increase in T3 concentrations, respectively. PFOA, PFNA, and PFDA were the predominant compounds in PFASs-FT3 associations, and the corresponding estimates were 0.11 (95\% Crl: 0.02, 0.19), - 0.17 (95\% Crl: - 0.28, - 0.07), and 0.12 (95\% Crl: $0.004,0.24) \mathrm{pmol} / \mathrm{L}$, respectively. A change in PFNA and PFOA concentrations from the 25th to 75th percentile was associated with a $-1.69(95 \% \mathrm{Crl}:-2.98,-0.41) \mu \mathrm{lU} / \mathrm{mL}$ decrease and a $1.51(95 \% \mathrm{Crl}: 0.48,2.55) \mu \mathrm{lU} / \mathrm{mL}$ increase in TSH concentrations. The associations of PFOA and PFNA with T3/FT3 were more pronounced in boys, while those with TSH were more pronounced in girls.

Conclusion: Our results suggest that prenatal exposure to multiple PFASs was associated with thyroid hormones in cord blood. However, individual PFAS had varied effects—differing in magnitude and direction—on fetal thyroid hormones.

Keywords: Perfluoroalkyl substances, Prenatal exposure, Thyroid hormones, Cord blood, Bayesian kernel machine regression, Prospective cohort study

\footnotetext{
* Correspondence: yuanwei11@yahoo.com

'Department of Social Medicine and Reproductive Epidemiology, National Health Commission Key Laboratory of Reproduction Regulation (Shanghai Institute of Planned Parenthood Research), Fudan University, \#779 Lao Hu Min Road, Shanghai 200032, China

Full list of author information is available at the end of the article
}

C C The Author(s). 2020 Open Access This article is licensed under a Creative Commons Attribution 4.0 International License, which permits use, sharing, adaptation, distribution and reproduction in any medium or format, as long as you give appropriate credit to the original author(s) and the source, provide a link to the Creative Commons licence, and indicate if changes were made. The images or other third party material in this article are included in the article's Creative Commons licence, unless indicated otherwise in a credit line to the material. If material is not included in the article's Creative Commons licence and your intended use is not permitted by statutory regulation or exceeds the permitted use, you will need to obtain permission directly from the copyright holder. To view a copy of this licence, visit http://creativecommons.org/licenses/by/4.0/. The Creative Commons Public Domain Dedication waiver (http://creativecommons.org/publicdomain/zero/1.0/) applies to the data made available in this article, unless otherwise stated in a credit line to the data. 


\section{Introduction}

Perfluoroalkyl substances (PFASs) are synthetic fluorinecontaining chemicals that have been extensively used in a range of industrial and commercial products during the past 60 years [1]. The general population is exposed to PFASs mainly through dietary intake of contaminated water and food, followed by indoor dust inhalation [2]. Certain PFASs, such as perfluorooctane sulfonate (PFOS), perfluorooctanoic acid (PFOA), perfluorohexane sulfonate (PFHxS), perfluorononanoic acid (PFNA), and perfluorodecanoic acid (PFDA), can be detected in $90 \%$ or more of the pregnant women in the US, Europe and Asia [3-7]. Since PFOS and PFOA were added to Annex $B$ of the Stockholm Convention on Persistent Organic Pollutants in 2009, PFAS production has been restricted in many European countries and the US. However, other long-chain PFASs and new alternatives are being manufactured in increasing volumes in some countries, including China $[8,9]$. PFASs are persistent in the environment due to their high resistance to degradation and long half-lives $[1,10]$. Therefore, the adverse effects of PFAS exposure continue to pose a public health concern. Since PFASs can easily cross the placental barrier and expose the fetus [11, 12], there is significant concern regarding the adverse effects of in utero PFAS exposure.

Toxicological studies have demonstrated that prenatal PFAS treatment can disrupt thyroid function in pregnant rats and pups, characterized by hypothyroidism [13-16]. Thyroid hormones (THs) play a critical role in normal fetal and child growth and neurodevelopment. Alterations in maternal and neonatal thyroid functions are associated with neurodevelopmental and cognitive deficits in children $[17,18]$. However, evidence regarding the effects of prenatal PFAS exposure on human fetal THs is inconsistent. Several studies have shown that maternal PFAS concentrations are associated with decreased fetal thyroxine (T4) and/or triiodothyronine (T3) concentrations accompanied by increased or unchanged thyroid stimulating hormone (TSH) concentrations [12, 19, 20]. Some studies have otherwise reported associations of cord PFASs with increased T4 and/or T3 concentrations accompanied by decreased or unchanged TSH $[11,21-$ 23]. Most of the associations are observed in a sexspecific manner [19, 21-24]. In addition, most prior studies examined PFASs as individual chemicals although the actual exposure involves a mixture of highly correlated PFASs simultaneously, thus leaving the residual confounding effect of co-exposure uncontrolled. Multi-pollutant models are needed to adequately consider the complexity of multiple PFAS exposure, addressing highly correlated compounds and their potential interaction.

Our previous study based on the Shanghai-Minhang Birth Cohort Study in China reported that PFAS concentrations in pregnant women, especially PFOS and PFOA (geometric mean: 10.78 and $19.62 \mathrm{ng} / \mathrm{ml}$, respectively), were much higher than those in the US and other Asian and European countries during a comparable time period [3]. Thus, in this study, we examined the associations between relatively higher concentrations of maternal plasma PFASs and thyroid hormone concentrations in cord blood, and evaluated the potential effect modification by infant sex. Moreover, we used the Bayesian kernel machine regression model, a recently developed multi-pollutant model, to assess the overall and singleexposure effects of the PFAS mixture on fetal THs.

\section{Methods}

\section{Study design and participants}

The present study was based on the Shanghai-Minhang Birth Cohort Study (S-MBCS), an ongoing prospective birth cohort study, which aims to determine the distributions of maternal environmental exposure and their effects on pregnant women and their children. Pregnant women at 12-16 gestational weeks were enrolled in the S-MBCS between April and December of 2012 during their first prenatal visit at the Maternal and Child Hospital of Minhang District in Shanghai. Participant recruitment criteria have been previously described in detail $[3,25]$. Briefly, pregnant women were recruited if they had no history of major chronic diseases diagnosed by a physician, planned to deliver in this specific hospital, and were willing to attend the specified interviews during pregnancy and after delivery. At enrollment, pregnant women were interviewed face-to-face by trained interviewers using a structured questionnaire. Information about demographic characteristics and lifestyle, maternal parity, dietary intake, vitamin use, and disease history was obtained.

A total of 1292 pregnant women agreed to participate in the study (participation rate: 77.4\%), 981 of whom had PFAS measurements from blood samples collected at enrollment. There were 1225 singleton live births at delivery, from which 615 cord blood samples were collected due to practical reasons. We further selected 348 cord blood samples for TH measurements under the conditions that the child had complete delivery information, had cord plasma volume $\geq 1 \mathrm{ml}$, and had at least one home visit at 12 or 48 months of age subject to limited funding. Finally, 308 mother-infant pairs had both maternal plasma PFAS concentrations and TH concentrations in cord blood measured. After excluding eight infants whose mothers reported prior or current diagnoses of thyroid-related disease/medication use at enrollment, 300 mother-infant pairs were included in the present study (Fig. 1).

The study protocol was approved by the Shanghai Institute of Planned Parenthood Research. All participants 


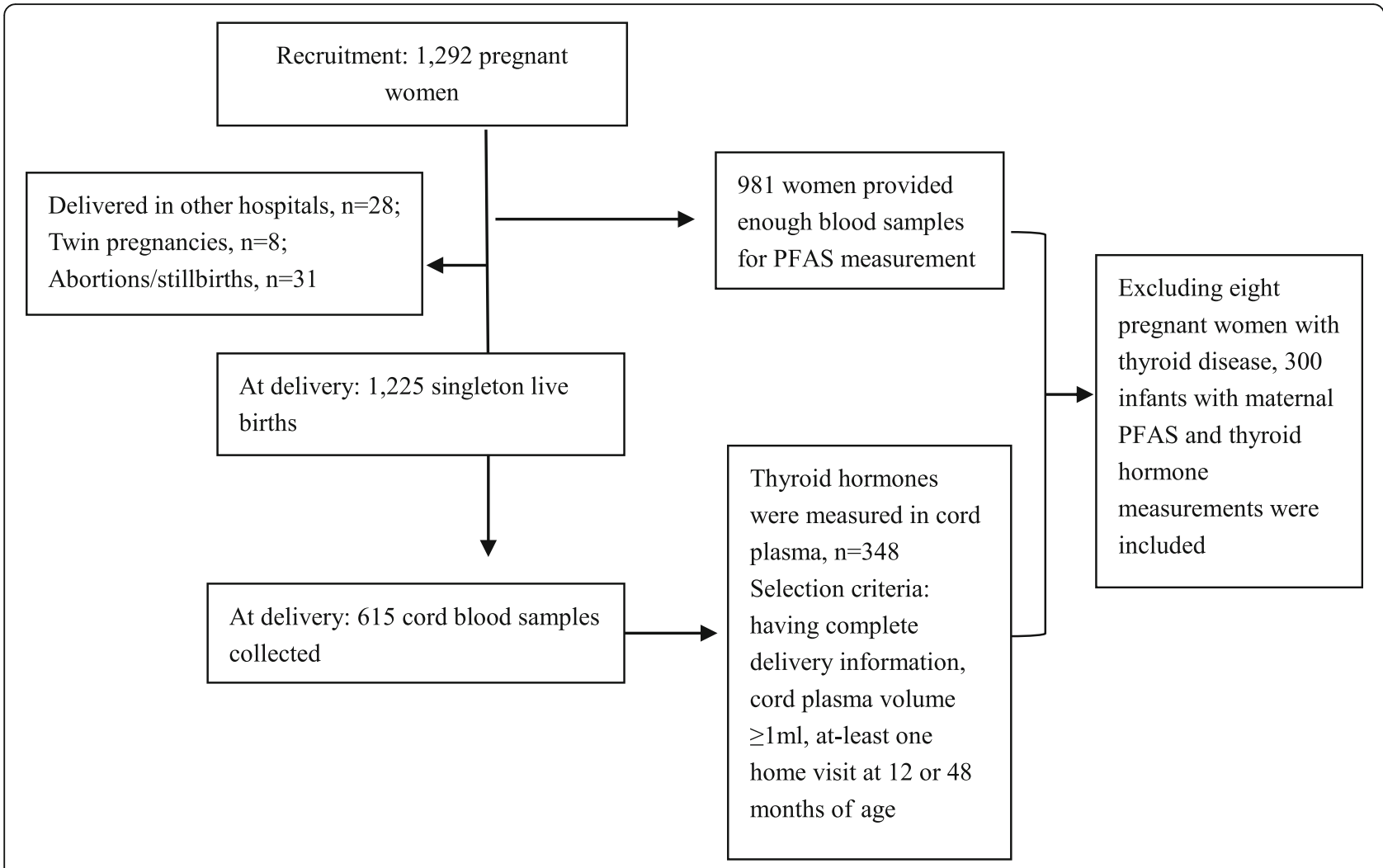

Fig. 1 Flow chart of study population selection in the Shanghai-Minhang Birth Cohort Study, 2012

provided written informed consent for themselves and their children at enrollment and subsequent visits.

\section{Maternal PFAS measurements}

One single blood sample was collected from each pregnant woman at 12-16 gestational weeks. After centrifugation, plasma samples were separated and stored at $80{ }^{\circ} \mathrm{C}$ until shipment to the Center for Disease Control and Prevention in Hubei Province for PFAS measurements. The concentrations of eleven PFASs in maternal plasma were measured using high-performance liquid chromatography coupled with tandem mass spectrometry (HPLC-MS-MS; Agilent Technologies Inc., USA). A detailed protocol for sample preparation, measurement, and limits of detection (LODs) has been previously described [3]. In the present study, we included eight PFASs that were detected in more than $80 \%$ of plasma samples including PFHxS, PFOS, PFOA, PFNA, PFDA, perfluoroundecanoic acid (PFUdA), perfluorododecanoic acid (PFDoA), and perfluorotridecanoic acid (PFTrDA). Concentrations below the LOD were replaced by $\mathrm{LOD} / \sqrt{2}$ in the analyses.

\section{Thyroid hormone assays in cord plasma}

Cord blood sample was collected from an umbilical vein immediately after delivery. Plasma samples were separated and stored at $-80^{\circ} \mathrm{C}$. TH concentrations in cord plasma were measured at the clinical laboratory in the affiliated hospital of Shanghai Institute of Planned Parenthood Research. Concentrations of T3, free triiodothyronine (FT3), T4, free thyroxine (FT4), and TSH in cord plasma were measured by electrochemiluminescence immunoassay (ECLIA) with kits obtained from Roche Cobas e601 analyzer, as described previously [26]. The analytical sensitivities of T3, T4, FT3, FT4, and $\mathrm{TSH}$ were $0.3 \mathrm{nmol} / \mathrm{L}, 5.4 \mathrm{nmol} / \mathrm{L}, 0.4 \mathrm{pmol} / \mathrm{L}, 0.3 \mathrm{pmol} /$ $\mathrm{L}$, and $0.27 \mu \mathrm{IU} / \mathrm{mL}$, respectively. The examiner was blinded to the infant's exposure status at the time of assessment.

\section{Statistical analyses}

We described and compared the demographic characteristics of the mother-infant pairs included and excluded from this study. We then described the distributions of maternal plasma PFAS concentrations and TH concentrations in cord plasma using percentiles and geometric means (GM). PFAS concentrations were natural log (ln) transformed to approximate the normal distribution for further analyses. The correlations between the pairs of ln-transformed PFAS concentrations and between the pairs of thyroid hormones were examined using Pearson correlation. 
We first used the generalized additive model (GAM) to examine the non-linearity ( $p$-value $<0.05$ ) of the associations between maternal PFAS concentrations and THs in cord plasma, and some of the associations showed non-linearity (Supplemental Figure S1). We then conducted multiple linear regressions to examine the associations of THs with ln-transformed PFAS concentrations and PFAS tertiles, respectively. The variables that were risk factors for maternal PFAS or TH concentrations based on previous literature and our data were considered potential confounders, and a directed acyclic graph was used to identify the covariates (Supplemental Figure S2). The following variables were adjusted for in the final analyses: maternal age at delivery, prepregnancy body mass index (BMI: < 18.5, 18.5-23.9, and $\geq 24 \mathrm{~kg} / \mathrm{m}^{2}$ ), education (high school or less, college or higher), parity $(1, \geq 2)$, gestational age $(<37$ or $\geq 37$ weeks), type of delivery (vaginal delivery, cesarean section), maternal passive smoking during pregnancy (yes, no), and maternal folic acid supplement during the first trimester (yes, no). Furthermore, paternal drinking during 3 months before pregnancy (yes, no) and infant sex (boy, girl) are strong predictors and they were included in the models to reduce imprecision. The information on maternal delivery date (to calculate age at delivery), type of delivery, gestational age, and infant sex was retrieved from medical birth records, while other information including maternal birth date, pre-pregnant weight and height, education, passive smoking during pregnancy, maternal folic acid supplement, and paternal drinking 3 months before pregnancy was obtained from the structured questionnaire. Maternal folic acid supplement was defined as taking the folic acid tablet regularly once a day. Paternal drinking was defined as paternal consumption of alcohol at least once a week in the last 3 months before conception and the information was reported by pregnant women at recruitment.

We further conducted a Bayesian kernel machine regression (BKMR) model, an approach for estimating the health effects of mixture exposure, to explore the overall effects of prenatal exposure to eight PFASs (ln-transformed concentrations) on each fetal $\mathrm{TH}$, and to identify important PFASs in association with each $\mathrm{TH}$, while adjusting for the same covariates as in the linear regression models. In addition, BKMR can assess potential interactions among multiple exposures. BKMR combines Bayesian and statistical learning methods to iteratively regress a response variable on a nonparametric term of exposure mixture components, through a Gaussian kernel function, that allows for non-linear and non-additive effects. Further, a hierarchical variable selection procedure within BKMR has been developed to account for the structure of the mixture and systematically handle highly correlated exposure $[27,28]$. We presented the graphical output generated by BKMR to show the overall and single-exposure effects of the PFAS mixture on each $\mathrm{TH}$. Figures of the overall effect show the point estimates and their 95\% credible intervals (95\% CrI) for the difference in each $\mathrm{TH}$ concentration holding concentrations of all eight PFASs at various quantiles (ranging from 0.25 to 0.75 ), as compared to fixing all PFAS concentrations at their 25th percentile. Figures of the singleexposure effect show the specific point estimates and their 95\% CrIs for the difference in each TH concentration for a change in individual PFAS concentrations between the 25th and 75th percentile, when holding the other PFASs at their 25th, 50th, and 75th percentiles, respectively. The relative importance of each PFAS was assessed using posterior inclusion probabilities (PIPs) of $>0.5$.

In the study, BKMR models were run in all participants, and then the analyses were stratified by infant sex due to its potential effect modification. We also stratified the analyses by type of delivery since it can influence FT3 and TSH concentrations [29]. To examine whether the associations between the PFAS mixture and THs were robust to the inclusion of additional co-exposed endocrine disrupting chemicals (EDCs), we fitted BKMR models by further including maternal urine bisphenol A (BPA) at recruitment and the sum of five polybrominated diphenyl ethers (Sum 5 PBDEs: BDE-28, - 47, - 99, -100 , and -153) in cord blood as co-exposures. BPA and PBDE measurements have been introduced previously $[26,30]$. We did not measure maternal iodine status, which is an important determinant of neonatal thyroid function. However, we collected the information on fish intake (iodine-rich food), and further adjusted for fish intake during the first trimester ( $\geq 3$ days/week, 1-2 days/week, and $<1$ day/week) in BKMR models to examine the robustness of the associations.

We used $\mathrm{R}$ software version 3.6.2 ( $\mathrm{R}$ Development Core Team) to conduct GAMs and BKMR analyses, and used SAS version 9.4 (SAS Institute Inc., Cary, NC, USA) to perform other statistical analyses. The statistical analyses were two-sided, with $p$-value $<0.05$ considered statistically significant.

\section{Results}

The characteristics of the included and excluded mother-infant pairs are presented in Table 1. The included mothers had a mean age of 27.5 years, most of whom were nulliparous (87.0\%), had normal prepregnant BMI $(73.9 \%)$, and attained college or higher education (77.3\%). About one-third of the included mothers reported a per capita household income of more than $8000 \mathrm{CNY} /$ month, and about $43 \%$ experienced passive smoking before conception. Among the included infants, $55.6 \%$ were male, and $48.3 \%$ were born 
via cesarean section. Only $1.7 \%$ of infants were born preterm. The included mother-infant pairs had similar characteristics to those excluded, except for a lower percentage of preterm births (Table 1).

PFOA was the dominant PFAS with the highest concentrations (GM: $19.41 \mathrm{ng} / \mathrm{mL}$ ), followed by PFOS (GM: $10.77 \mathrm{ng} / \mathrm{mL}$ ). GMs of PFHxS, PFDA, PFNA, PFUdA, PFDoA, and PFTrDA were 2.66, 2.15, 1.82, 1.61, 0.09, and $0.09 \mathrm{ng} / \mathrm{mL}$, respectively, (Table 2). The distributions of eight PFASs in the included participants were comparable with those excluded (Table S1). As in our previous study, the ln-transformed PFAS concentrations were moderately to highly correlated with each other (Pearson correlation coefficients $[r]=0.33-0.89$ ), except for the PFHxS-other compounds and PFOA-PFDoA/ PFTrDA relationships (Supplemental Table S2). The GMs of cord blood T3, FT3, T4, FT4, and TSH concentrations were $0.85 \mathrm{nmol} / \mathrm{L}, 1.77 \mathrm{pmol} / \mathrm{L}, 90.63$ $\mathrm{nmol} / \mathrm{L}, 14.1 \mathrm{pmol} / \mathrm{L}$, and $6.49 \mu \mathrm{IU} / \mathrm{mL}$, respectively (Table 2). The correlations between the $\mathrm{TH}$ hormones were stronger between T4 and FT4 $(r=0.82)$ and between T3 and FT3 $(r=0.79)$. There were moderate correlations between FT3 and T4/FT4 $(r=0.44 / 0.49)$ and between TSH and T4/FT4 $(r=0.39 / 0.24)$. There was no correlation between other pairs of TH hormones (Supplemental Table S3). Infants born via vaginal delivery had statistically significantly higher TSH concentrations but lower FT3 and FT4 concentrations than those born via cesarean section (Supplemental Table S4).

In linear regression models, ln-transformed concentrations of PFDoA, PFUdA, PFOS, PFNA, PFDA, and PFOA were associated with increased T3 concentrations [0.02 (95\% confidence interval $[\mathrm{CI}]: 0.00,0.04)$ to 0.07 $(95 \%$ CI: $0.02,0.11) \mathrm{nmol} / \mathrm{L}]$ in cord plasma after

Table 1 Characteristics of mother-infant pairs included and excluded in the study

\begin{tabular}{|c|c|c|c|}
\hline Variables $^{\mathbf{a}}$ & $\begin{array}{l}\text { Participants included } \\
(N=300) \\
\mathrm{n}(\%)\end{array}$ & $\begin{array}{l}\text { Participants excluded } \\
(N=925) \\
n(\%)\end{array}$ & $\begin{array}{l}P \text {-value of Student's t-test } \\
\text { or Chi-square test }\end{array}$ \\
\hline Maternal age at birth (years, mean \pm SD) & $27.5 \pm 3.5$ & $27.9 \pm 3.4$ & 0.0592 \\
\hline \multicolumn{4}{|l|}{ Maternal pre-pregnant BMI (kg/m²) } \\
\hline$<18.5$ & $63(21.4)$ & $181(19.9)$ & 0.8633 \\
\hline $18.5-24.9$ & $218(73.9)$ & $683(75.1)$ & \\
\hline$\geq 25.0$ & $14(4.7)$ & $45(5.0)$ & \\
\hline \multicolumn{4}{|l|}{ Parity } \\
\hline Nulliparous & $261(87.0)$ & $767(83.7)$ & 0.1744 \\
\hline Multiparous & $39(13.0)$ & $149(16.3)$ & \\
\hline \multicolumn{4}{|l|}{ Maternal education } \\
\hline High school or less & $68(22.7)$ & $226(24.5)$ & 0.5460 \\
\hline College or higher & $231(77.3)$ & $698(75.5)$ & \\
\hline \multicolumn{4}{|l|}{ Per capita household income (CNY/month) } \\
\hline$<4000$ & $66(22.1)$ & $187(20.5)$ & 0.2290 \\
\hline $4000-8000$ & $130(43.5)$ & $359(39.5)$ & \\
\hline$>8000$ & $103(34.4)$ & $364(40.0)$ & \\
\hline \multicolumn{4}{|l|}{ Maternal passive smoking before conception } \\
\hline No & $171(57.2)$ & $556(60.3)$ & 0.3406 \\
\hline Yes & $128(42.8)$ & $366(39.7)$ & \\
\hline \multicolumn{4}{|l|}{ Gestational age (weeks) } \\
\hline$\geq 37$ & $295(98.3)$ & $884(95.7)$ & 0.0332 \\
\hline$<37$ & $5(1.7)$ & $40(4.3)$ & \\
\hline \multicolumn{4}{|l|}{ Type of delivery } \\
\hline Vaginal & $154(51.7)$ & $368(46.5)$ & 0.1246 \\
\hline Cesarean section & $144(48.3)$ & $424(53.5)$ & \\
\hline \multicolumn{4}{|l|}{ Child's sex } \\
\hline Male & $167(55.6)$ & $500(54.2)$ & 0.6642 \\
\hline Female & $133(44.4)$ & $422(45.8)$ & \\
\hline
\end{tabular}

${ }^{\mathrm{a} S D}$ Standard deviation, BMI Body mass index, CNY Chinese Yuan 
Table 2 Descriptive statistics of PFAS concentrations in maternal plasma and thyroid hormone concentrations in cord plasma

\begin{tabular}{|c|c|c|c|c|c|c|c|}
\hline & \multirow{2}{*}{$\begin{array}{l}>\text { LOD } \\
\text { (\%) }\end{array}$} & \multicolumn{5}{|c|}{ Percentiles } & \multirow[t]{2}{*}{ GM (SD) } \\
\hline & & 5th & 25th & 50th & 75th & 95th & \\
\hline \multicolumn{8}{|c|}{ PFAS $(N=300, \mathrm{ng} / \mathrm{mL})$} \\
\hline PFHxS & 100 & 1.29 & 2.04 & 2.67 & 3.40 & 5.48 & $2.66(1.51)$ \\
\hline COS & 100 & 4.04 & 7.34 & 10.49 & 16.30 & 26.47 & $10.77(1.80)$ \\
\hline FOA & 100 & 9.17 & 14.49 & 19.38 & 26.80 & 38.00 & $19.41(1.56)$ \\
\hline NA & 100 & 0.84 & 1.38 & 1.80 & 2.51 & 3.88 & 1.8 \\
\hline EAA & 100 & 0.75 & 1.41 & 2.15 & 3.28 & 6.30 & $2.15(1.91)$ \\
\hline FUdA & 100 & 0.54 & 1.04 & 1.68 & 2.55 & 4.38 & $1.61(1.95)$ \\
\hline EDoA & 91.04 & 0 & 0.05 & 0.11 & 0.20 & 0.39 & $0.09(3.09)$ \\
\hline FTrDA & 88.93 & 0 & 0.04 & 1 & 0.18 & 0 & 95) \\
\hline \multicolumn{8}{|c|}{ Thyroid hormones $(N=300)$} \\
\hline (nmol/L) & 100 & 0.67 & 0.77 & 0.84 & 0.94 & 1.10 & 0.85 \\
\hline FT3 (pmol/L) & 98 & 1.34 & 1.53 & 1.76 & 2.00 & 2.42 & $1.77(1.21)$ \\
\hline $4(\mathrm{nmol} / \mathrm{L})$ & 100 & 49.30 & 77.67 & 93.10 & 109.90 & 143.65 & $90.63(1.36)$ \\
\hline FT4 (pmol/L) & 100 & 11.08 & 12.82 & 14.14 & 15.39 & 17.81 & $14.10(1.15)$ \\
\hline $\mathrm{TSH}(\mu \mathrm{IU} / \mathrm{mL})$ & 100 & 2.24 & 4.32 & 6.50 & 9.72 & 18.97 & $6.49(1.87)$ \\
\hline
\end{tabular}

LOD Limit of detection, GM Geometric mean, SD Standard deviation, $P F H \times S$ Perfluorohexane sulfonic acid, PFOS Perfluorooctane sulfonate, PFOA Perfluorooctanoic acid, PFNA Perfluorononanoic acid, PFDA Perfluorodecanoic acid, PFUdA Perfluoroundecanoic acid, PFDoA Perfluorododecanoic acid, PFTrDA Perfluorotridecanoic acid, PFTeDA Perfluorotetradecanoic acid, PFHXDA Perfluorohexadecanoic acid, PFDS Perfluorodecane sulfonate; $T_{3}$, total triiodothyronine, $T_{4}$ Total thyroxine, $F T_{3}$ Free triiodothyronine, $F T_{4}$ Free thyroxin, TSH Thyroid stimulating hormone

adjusting for covariates. Meanwhile, per ln-unit increase in PFOS, PFOA, and PFDA concentrations was associated with a 0.09 (95\%CI: 0.02, 0.16), 0.13 (95\%CI: 0.03, 0.23 ), and 0.08 (95\%CI: $0.01,0.15) \mathrm{pmol} / \mathrm{L}$ increase in FT3 concentrations, respectively, (Table 3). Lntransformed concentrations of PFNA, PFDA, and PFUdA were associated with decreased T4 [-7.81 (95\%CI: - 14.38, - 1.24), - 5.07 (95\%CI: - 9.78, - 0.37), and -4.90 (95\%CI: $-9.44,-0.35) \mathrm{nmol} / \mathrm{L}$, respectively] and TSH concentrations [- 1.61 (95\% CI: $-2.90,-0.31)$, - 0.96 (95\% CI: - 1.89, - 0.03), and - 1.06 (95\%CI: - 1.95, - 0.16) $\mu \mathrm{IU} / \mathrm{mL}$, respectively]. No association between PFASs and FT4 concentrations was observed (Table 3). The linear regression models using categorized PFAS concentrations showed similar results to those in the analyses above in general (Supplemental Table S5). However, the associations of PFHxS in middle tertile with increased T3, of PFDoA in middle tertile with increased FT3, of PFOA in middle tertile with decreased T4/FT4, and of PFTrDA in high tertile with decreased $\mathrm{TSH}$, were observed.

The BKMR model could assess the overall effects of the PFAS mixture on THs when all of the compounds are held at specified quantiles. As indicated in Fig. 2a, higher PFAS mixture concentrations were associated with increased T3 concentrations, such as that compared with the 25th percentile, the 75th percentile of the PFAS mixture was associated with a 0.074 (95\% CrI: 0.037, 0.146) $\mathrm{nmol} / \mathrm{L}$ increase in T3 concentrations. There were suggestive associations between the PFAS mixture and increased FT3 concentrations (estimate at the 75th percentile: 0.095 [95\%CrI: $-0.005,0.195] \mathrm{pmol} / \mathrm{L}$; Fig. 2b). There was a pattern of decreased T4 and FT4 concentrations associated with the PFAS mixture, however, with all CrIs across null (Fig. 2c and d). No association was observed between the PFAS mixture and TSH (Fig. 2e).

Figure 3 presents the single-exposure effects of individual PFASs on THs when holding the other compounds at the 25th, 50th, and 75th percentiles, respectively. In PFASs-T3 associations, PFDA had the highest PIP (0.6868), followed by PFUdA (0.3142) and PFOA (0.2116), respectively. When holding all other PFASs at the median concentrations, a change in PFDA, PFUdA and PFOA concentrations from the 25th to 75th percentile was associated with a $0.04(95 \% \mathrm{CrI}:-0.01$, 0.09 ), 0.02 (95\%CrI: $-0.03,0.07)$, and 0.03 (95\%CrI: $0.001,0.06) \mathrm{nmol} / \mathrm{L}$ increase in T3, respectively, although without statistical significance (Fig. 3a). In PFASs-FT3 associations, PFOA, PFNA, and PFDA were the predominant compounds with PIPs of 0.94, 0.781, and 0.7448 , respectively. The corresponding FT3 concentration changes associated with a change in PFOA, PFNA and PFDA concentrations from the 25th to 75th percentile were 0.11 (95\%CrI: $0.02,0.19), \quad-0.17$ (95\%CrI: $-0.28,-0.07)$, and 0.12 (95\%CrI: $-0.004,0.24)$ $\mathrm{pmol} / \mathrm{L}$, respectively (Fig. 3b). Although no individual compound showed PIP $>0.5$ in PFASs-TSH associations, PFNA showed the highest PIP (0.4072), followed by PFOA (0.2922). A change in PFNA and PFOA concentrations from the 25th to 75th percentile was associated with a -1.69 (95\%CrI: - 2.98, - 0.41) $\mu \mathrm{IU} / \mathrm{mL}$ decrease and a $1.51(95 \% \mathrm{CrI}: 0.48,2.55) \mu \mathrm{IU} / \mathrm{mL}$ increase in TSH (Fig. 3e). BKMR analyses did not identify a single, large contributor to the effects on T4 (all PIPs < 0.2) and FT4 concentrations (all PIPs < 0.04; Fig. 3c and d). The effect estimates of PFOA on T3 and FT3 showed shifts in magnitude in the same direction when the other compounds were held at varied quantiles $(0.25,0.50$, and 0.75), and those of PFDoA on FT3 showed changes in direction. We further calculated the interactive effect (Supplemental Figure S3), which showed that only the interaction of PFDoA with other PFASs was statistically significant. Most of the associations with T3, FT3, and TSH in BKMR models were similar to those observed in linear regression models. However, BKMR analyses showed that there were associations between PFNA and decreased FT3, and between PFOA and increased TSH, 


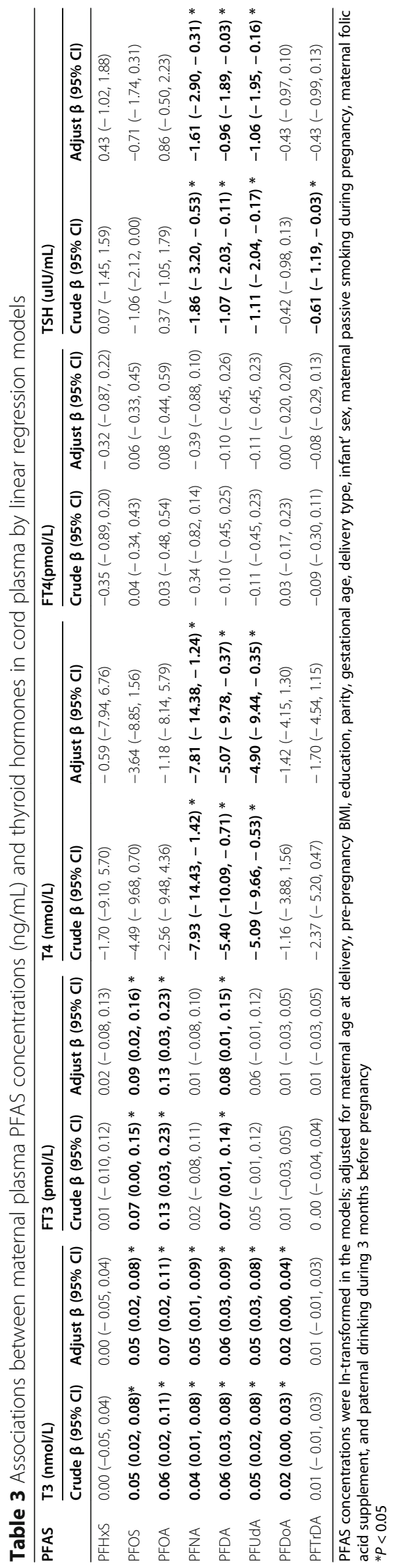


which was not observed in linear regression models. In contrast, some associations shown in linear regression models did not exist in BKMR analyses. Specifically, PFOS, PFNA, and PFDoA contributed much less to the associations with T3 (PIPs $\leq 0.025)$. Similarly, the associations between PFOS and FT3 (PIP: 0.037), between PFNA/PFDA/PFUdA and T4 (PIPs < 0.2), and between PFDA/PFUdA and TSH (PIPs $<0.12)$ attenuated significantly in BKMR models.

Figure 4 and Supplemental Figure S4 and S5 present the overall and single-exposure effects of the PFAS mixture on THs, stratified by infant sex. Overall, similar patterns to the pooled gender analyses were observed in each stratum. However, the statistically significant associations between the PFAS mixture and increased T3 concentrations were observed only in boys (Fig. 4a and b). Associations of PFOA and PFNA with T3/FT3 observed in the pooled gender analyses were more pronounced in boys, whereas their associations with TSH were more pronounced in girls (Fig. 4c and d). When the analyses were stratified by delivery type, patterns similar to the pooled analyses were also observed in each stratum in general (Supplemental Figure S6 and S7). However, the associations between the PFAS mixture and T3 were strengthened in infants born via cesarean section with the CrIs ranging from 0.02 (95\%CrI: 0.01, $0.03) \mathrm{nmol} / \mathrm{L}$ at the 30th percentile of the PFAS mixture to 0.09 (95\% CrI: $0.04,0.14) \mathrm{nmol} / \mathrm{L}$ at the 75 th percentile, compared with the 25th percentile. Other estimates in the overall effect analyses were across null (Supplemental Figure S6). For single-exposure effects, the associations of PFDA and PFOA with T3 were more pronounced in infants born via caesarean section, while the associations of PFNA with FT3 and of PFOA and PFNA with TSH were mainly observed in infants born via vaginal delivery (Supplemental Figure S7). After further including BPA and Sum ${ }_{5}$ PBDEs as co-exposures in BKMR models, the effects of individual PFASs on THs remained similar in all infants (Supplemental Figure S8). Further adjusting for fish intake during the first

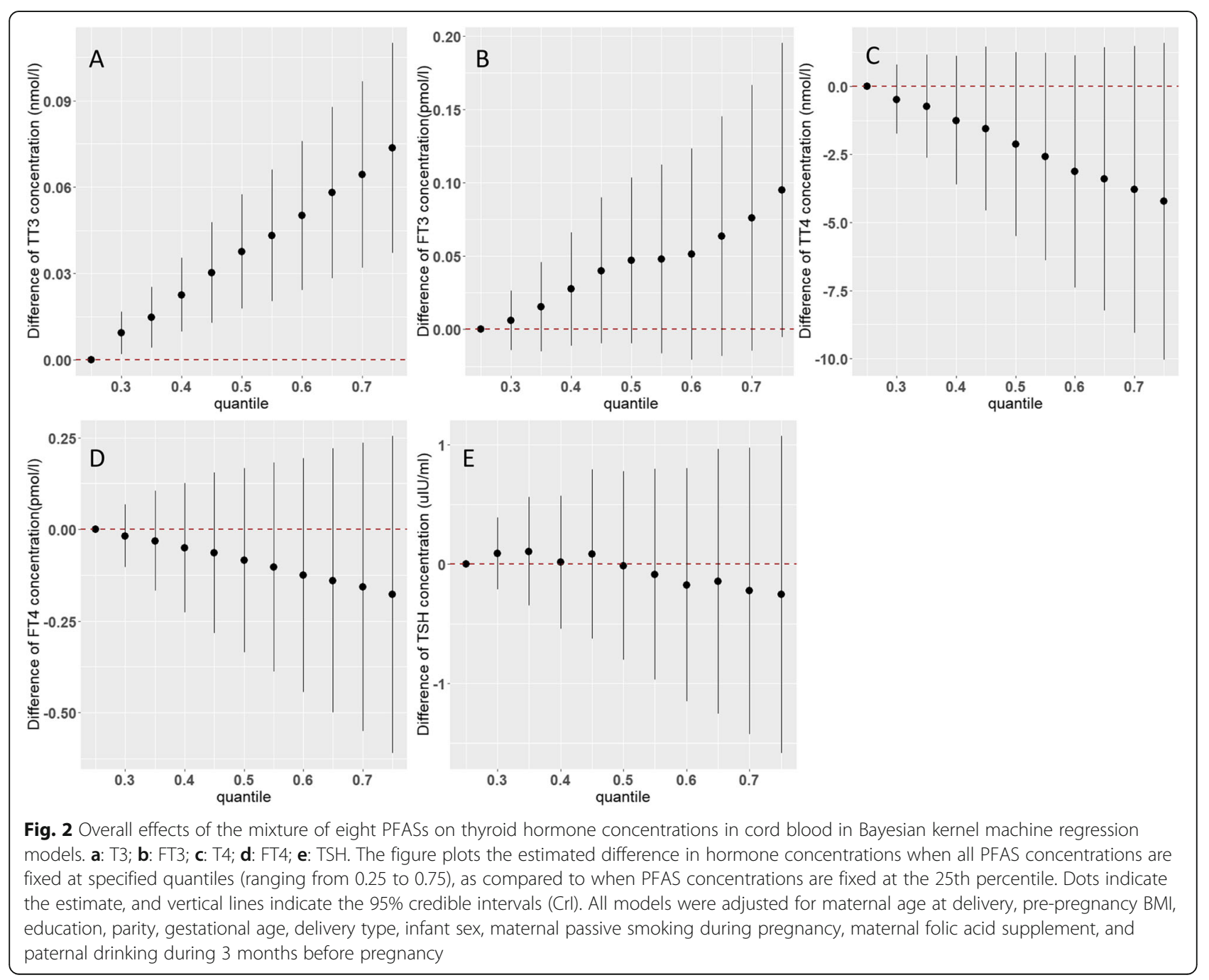




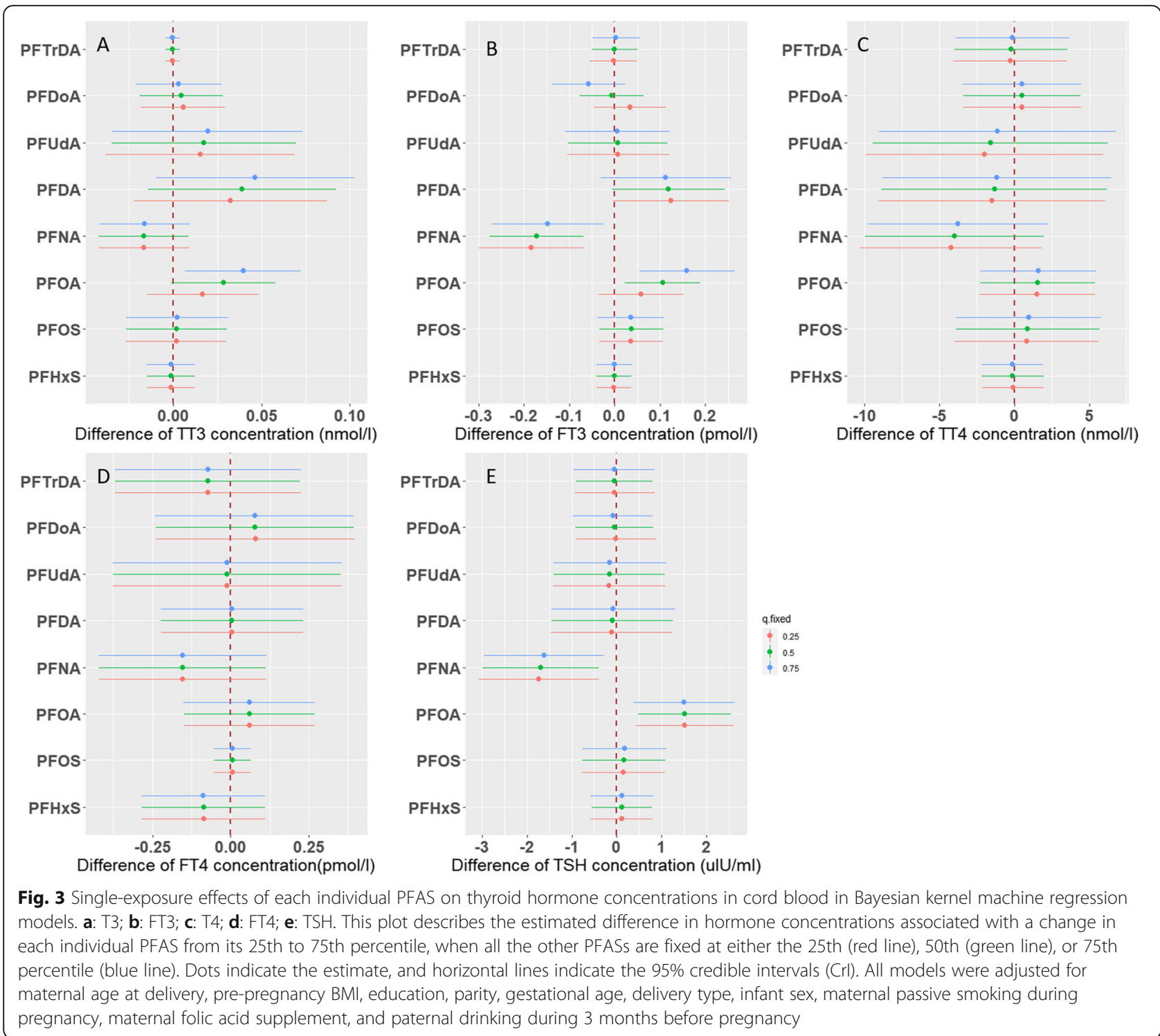

trimester in BKMR models did not change the observed associations (data not shown).

\section{Discussion}

In the study, we examined the overall and individual effects of maternal exposure to eight PFASs on TH concentrations in cord plasma when accounting for the correlations between multiple PFASs using BKMR models, and also examined the individual effects of eight PFASs using linear regression. In BKMR models, the PFAS mixture was associated with increased T3 and FT3 concentrations. For individual PFAS-hormone associations, maternal PFOA, PFDA, and PFUdA exposure was associated with increased T3 and FT3 (not for PFUdA) concentrations, and maternal PFNA exposure was associated with decreased TSH concentrations in both BKMR and linear regression models. BKMR models further identified that there were PFNA association with decreased FT3 concentrations and PFOA association with increased TSH concentrations. The associations of PFOA and PFNA with T3/FT3 were more pronounced in boys, while their associations with TSH were more pronounced in girls.

The observed associations from linear regression models of maternal PFOS, PFNA, and PFDoA exposure with increased T3 concentrations, PFOS and PFUdA with increased FT3 concentrations, PFNA, PFDA and PFUdA with decreased T4 concentrations, and PFDA and PFUdA with decreased TSH concentrations were not observed in BKMR models. These associations are possibly spurious because linear regression models could not deal with either high correlations between multiple PFASs or non-linear dose-responses that may bias the associations. 


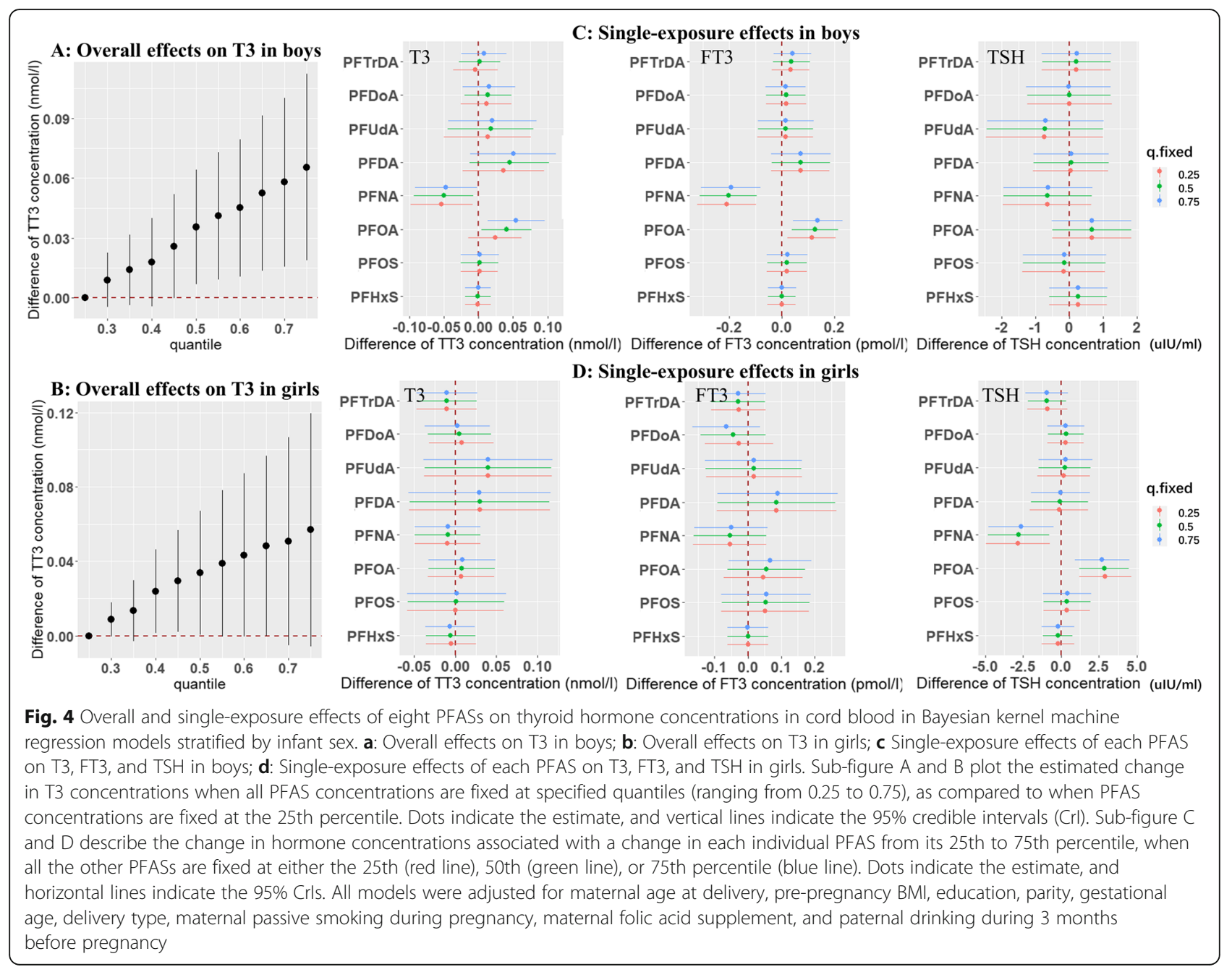

Our findings are not consistent with reports from rodent studies, which tend to report decreased T4 and unchanged T3/TSH in rat pups following prenatal PFAS exposure $[13,14,16]$. However, studies in zebrafish have reported increased T3 and unchanged T4 in the $F(0)$ and $F(1)$ generations following PFAS exposure [31, 32]. Interspecies differences and exposure doses in most animal studies that are significantly higher than those experienced by the human population may make it difficult to compare the effects of PFASs on thyroid function between animals and humans.

Only a few studies have used multi-pollutant models to address the correlations between multiple PFASs when examining their effects on THs in infants. Aimuzi et al's study [21] used sparse partial least squares regression to identify the dominant PFASs related to FT4, FT3, and TSH in cord blood, while the model did not estimate the joint effects of the PFAS mixture. Two studies from the US $[33,34]$ also used BKMR to assess the overall effects of prenatal PFAS mixture exposure on neonatal THs. The study based on the Project Viva cohort [33] showed a statistically non-significant inverse association between the PFAS mixture and lower T4 concentrations (T3 and FT3 not measured) in heel stick blood and no association with TSH, which is consistent with our findings. However, the study based on the HOME study [34] did not find any association between the PFAS mixture (PFOA, PFOS, PFNA, and PFHxS) and any $\mathrm{TH}$ in cord blood. For the single exposure effects of PFASs, our results were partially consistent with those of previous epidemiological studies. In this study, maternal PFAS exposure was associated with higher FT3 and T3 concentrations, which is consistent with three previous studies where maternal PFDA exposure was associated with higher FT3 among girls born via vaginal delivery [24], and cord blood PFDA and PFUdA were associated with higher $\mathrm{T} 3$ concentrations $[22,35]$. In addition, our finding that maternal PFNA was associated with decreased cord TSH is consistent with three previous studies [21, 22, 36], and that of maternal PFOA being associated with increased TSH is consistent with Kim's study [12]. Other studies that have examined 
PFAS exposure and TH concentrations tended to report that PFAS exposure was associated with decreased T4 (or FT4) and/or T3 with different compounds showing statistical significance [11, 19, 20, 34, 35].

We should bear in mind that it is quite difficult to directly compare our study with previous studies, due to differences in the timing and matrix of PFAS measurements (maternal blood at first, second, or third trimester vs. cord blood), measured PFASs and their concentrations, measured TH types and matrix (type: five THs vs. only T4/TSH measured, T4, T3, and TSH measured; matrix: cord blood vs. neonatal heel stick blood), characteristics of the study population (all infants vs. born via vaginal delivery or pre-labor cesarean section), and models used for analyses. Similar to another study conducted in Shanghai [21] but different from most previous studies, PFOA, but not PFOS, was the predominant compound in our study, and its concentration was much higher (median: 19.4 vs. $2.0-5.6 \mathrm{ng} / \mathrm{mL}$ ) than previously reported $[11,19,20,24]$. This may partly explain why associations between PFOA and THs were observed in this study. In addition, $\mathrm{TH}$ concentrations measured from cord blood and neonatal heel stick may be different since the stress during birth could cause TSH surge in cord blood. As shown in this study and previous literature [29], infants born via vaginal delivery had statistically significant higher TSH and lower FT3 concentrations than those born via cesarean section. Further stratified analyses by delivery mode showed that the PFAS-T3 associations were more pronounced in infants born via cesarean section, while PFAS-FT3/TSH associations were more pronounced in those born via vaginal delivery. Thus, the methodological difference in $\mathrm{TH}$ measurement and the characteristics of the population may lead to the difference in PFAS-TH associations to some extent. In addition, BKMR in the study included eight PFASs simultaneously and allowed the disentanglement of the independent effects of each compound among the co-exposed and highly correlated PFAS mixture. Most previous studies used conventional linear regression in a one-at-a-time approach without considering the high correlations between PFASs. The application of varied models may produce a different result, as shown in this study.

The underlying mechanism by which PFAS exposure disrupts thyroid homeostasis is still not clear. Toxicological studies have suggested several potential mechanisms. PFASs have a competitive binding ability with $\mathrm{TH}$ transport protein transthyretin [37], may enhance hepatic uptake and metabolism of $\mathrm{T} 4$ via the upregulation of uridine diphosphoglucuronosyl transferase 1A1 and hepatic organic anion transporter [38, 39], and increase the conversion of $\mathrm{T} 4$ to $\mathrm{T} 3$ via the upregulation of type 1 deiodinase [38]. These mechanisms may contribute to increased $\mathrm{T} 3$ and lower $\mathrm{T} 4$ concentrations following maternal PFAS exposure. However, the mechanisms could not explain all the findings since PFNA and PFOA were associated with T3, FT3, and TSH in opposite directions. Furthermore, most of the toxicological studies were based on PFOS exposure, while our study did not find associations between PFOS and THs. Some toxicological studies have suggested that individual PFASs may have different potency and mechanisms for exerting their disrupting effects, and their effects on THs need to be further clarified.

BKMR models suggested sex-specific effects of PFASs on THs, manifesting with more pronounced associations of PFOA and PFNA with T3/FT3 in boys and with TSH in girls. Sex-specific effects have also been observed in previous studies [21, 22]. Sexual hormones may influence $\mathrm{TH}$ concentrations by increasing serum thyroxinebinding globulin concentrations [40]. PFASs can affect estrogen and androgen receptor transactivity [41], interfere with steroidogenesis, and inhibit testosterone release from Leydig cells [42], leading to changes in estradiol (E2) and testosterone (T) concentrations. In humans, prenatal PFAS exposure is associated with decreased cord T/E2, progesterone, and inhibin B, and with increased E2 concentrations sex-specifically [43]. We speculated that affected sexual hormones might further influence thyroid homeostasis sex-specifically. However, the exact underlying mechanism has not yet been elucidated.

The major strength of the study was the prospective design that could provide potential causality of the associations between prenatal PFAS exposure and fetal THs. In addition, we applied multi-pollutant models of a BKMR approach to estimate the overall, single-exposure, and interactive effects of multiple PFASs on THs, addressing the high correlation of the PFAS mixture and the potential non-linear dose-responses.

The findings should also be interpreted in light of certain limitations. First, the study included a limited number of subjects $(24 \%)$ from the S-MBCS cohort, mainly because quite a few cord blood samples were not collected due to practical reasons, and samples were further selected for $\mathrm{TH}$ measurement due to limited funding. It is less likely that these reasons for attrition are associated with both maternal PFAS concentrations and cord blood TH concentrations. Furthermore, compared with those excluded, the included mother-infant pairs had similar characteristics except for a slightly higher proportion of preterm births, and similar PFAS distributions. The attrition seemed to be non-differential, which alleviated our concern regarding selection bias. Second, we measured $\mathrm{TH}$ concentrations in cord blood rather than neonatal blood. A neonatal TSH surge occurs at delivery caused by intrapartum fetal stress, and neonates born vaginally had higher TSH and lower FT3 
concentrations in cord blood than those born via cesarean section. Therefore, we stratified the analyses by delivery type to reduce the influence due to TSH surge, especially during vaginal delivery. Third, we could not control potential confounding effects of other unmeasured EDCs co-exposed with PFASs, if they are correlated with PFASs. In addition, we did not measure maternal iodine status, which is an important determinant of neonatal thyroid function. However, we further adjusted for fish intake (one kind of iodine-rich food) in BKMR models, and the results remained similar. At last, although we measured the concentrations of five THs, we did not measure cord blood concentrations of thyroid transport proteins and thyroid antibodies; thus, we could not address the overall picture of associations related to fetal thyroid hormone homeostasis.

\section{Conclusion}

In this study, prenatal exposure to the PFAS mixture was associated with increased T3 and FT3 concentrations in cord blood. In terms of the predominant compounds, maternal PFOA, PFDA, and PFUdA exposure was associated with increased T3 and FT3 (not for PFUdA), accompanied by PFOA associated with increased TSH. In contrast, PFNA was associated with decreased FT3 and TSH concentrations. The effects of PFOA and PFNA on THs may be sex-specific, with more pronounced effects on FT3/T3 in boys and TSH in girls. These findings suggest that prenatal co-exposure to multiple PFASs may affect fetal thyroid hormones, and individual PFASs have varied effects-differing in magnitude and direction—on fetal thyroid hormones.

\section{Supplementary Information}

Supplementary information accompanies this paper at https://doi.org/10. 1186/s12940-020-00679-7.

Additional file 1: Figure S1 Adjusted generalized additive model plots of In-transformed PFAS concentrations with thyroid hormone concentrations. Figure S2 The causal network between maternal plasma PFAS concentrations and thyroid hormones (TH) in cord plasma, presented in a directed acyclic graph. Figure $\mathbf{S} 3$ Interactive effects of each PFAS with other remaining compounds in the associations of maternal PFAS concentrations with total triiodothyronine (T3; A) and free T3 (FT3; B) concentrations. Figure S4 Overall effects of the mixture of eight PFASs on thyroid hormone concentrations in cord blood in Bayesian kernel machine regression models stratified by infant sex. Figure $\mathbf{S 5}$ Singleexposure effects of each individual PFAS on thyroid hormone concentrations in cord blood in Bayesian kernel machine regression models stratified by infant sex. Figure $\mathbf{S 6}$ Overall effects of the mixture of eight PFASs on thyroid hormone concentrations in cord blood in Bayesian kernel machine regression models stratified by type of delivery. Figure S7 Singleexposure effects of each individual PFAS on thyroid hormone concentrations in cord blood in Bayesian kernel machine regression models stratified by type of delivery. Figure $\mathbf{S 8}$ Single-exposure effects of each individual PFAS, bisphenol A (BPA), and polybrominated diphenyl ethers (PBDEs) on thyroid hormone concentrations in cord blood in Bayesian kernel machine regression models. Table $\mathbf{S 1}$ Comparisons of maternal plasma PFAS concentrations between mother-infant pairs included and excluded from the present study using Mann-Whitney U test. Table S2 Pearson correlation coefficients between pairs of In-transformed PFAS concentrations $(\mathrm{ng} / \mathrm{mL})$ in maternal plasma. Table S3 Pearson correlation coefficients between pairs of thyroid hormones in 300 cord plasma samples. Table $\mathbf{S} \mathbf{4}$ Thyroid hormone concentrations in cord plasma among infants born via vaginal delivery or caesarean section. Table S5 Associations between maternal plasma PFAS concentrations (tertile, $\mathrm{ng} / \mathrm{mL}$ ) and thyroid hormones in cord plasma by linear regression models $(n=280)$.

\section{Abbreviations}

BKMR: Bayesian kernel machine regression; BMl: Body mass index (kg/m2); Cl: Confidence interval; Crl: Credible Interval; FT3: Free triiodothyronine;

FT4: Free thyroxine; GAM: Generalized additive model; GM: Geometric means; LODs: Limits of detection; PFASs: Perfluoroalkyl substances;

PFDA: Perfluorodecanoic acid; PFDoA: Perfluorododecanoic acid; PFHXS: Perfluorohexane sulfonate; PFNA: Perfluorononanoic acid; PFOA: Perfluorooctanoic acid; PFOS: Perfluorooctane sulfonate;

PFTrDA: Perfluorotridecanoic acid; PFUdA: Perfluoroundecanoic acid; PIPs: Posterior inclusion probabilities; S-MBCS: Shanghai-Minhang Birth Cohort Study; T3: Total triiodothyronine; T4: Total thyroxine; THs: Thyroid hormones; TSH: Thyroid stimulating hormone

\section{Acknowledgements}

We thank the field investigators involved in the survey for their efforts in data collection and all participants in the cohort.

\section{Authors' contributions}

WY, HL, and MM designed the study. HL analyzed the data and drafted the manuscript. ZL and YT contributed to the data analysis and interpretation of data. YZ and SW conducted measurements. YC and XS performed field investigation. All authors contributed in drafting and editing the manuscript and approved the final manuscript.

\section{Funding}

This work was supported by grants from the National Key Research and Development Program of China (2018YFC1002801; 2016YFC1000505), the Science and Technology Commission of Shanghai Municipality (20ZR1448000, 16ZR1430100), Innovation-oriented Science and Technology Grant from NHC Key Laboratory of Reproduction Regulation (CX2017-06).

\section{Availability of data and materials}

The datasets used in the current study are available from the corresponding authors on reasonable request.

\section{Ethics approval and consent to participate}

The study was approved by the ethical review committee of Shanghai Institute of Planned Parenthood Research (SIPPR). The study was conducted according to the Declaration of Helsinki. All mothers gave their written informed consent for themselves and their children before enrollment and at each follow-up.

\section{Consent for publication}

Not applicable.

\section{Competing interests}

The authors declare that they have no competing financial interests.

\section{Author details}

${ }^{1}$ Department of Social Medicine and Reproductive Epidemiology, National Health Commission Key Laboratory of Reproduction Regulation (Shanghai Institute of Planned Parenthood Research), Fudan University, \#779 Lao Hu Min Road, Shanghai 200032, China. ${ }^{2}$ National Management Office of Neonatal Screening Project for CHD, Children's Hospital of Fudan University, 399 Wanyuan Road, Shanghai 201102, China. ${ }^{3}$ National Reference Laboratory of Dioxin, Institute of Health Inspection and Detection, Hubei Provincial Academy of Preventive Medicine, Hubei Provincial Center for Disease Control and Prevention, \#6 Zhuo Daoquan North Road, Wuhan 430079, China. 
Received: 7 August 2020 Accepted: 13 November 2020 Published online: 26 November 2020

\section{References}

1. Lindstrom AB, Strynar MJ, Libelo EL. Polyfluorinated compounds: past, present, and future. Environ Sci Technol. 2011;45(19):7954-61.

2. Fromme $H$, Tittlemier SA, Volkel W, Wilhelm M, Twardella D. Perfluorinated compounds--exposure assessment for the general population in Western countries. Int J Hyg Environ Health. 2009;212(3):239-70.

3. Tian Y, Zhou Y, Miao M, Wang Z, Yuan W, Liu X, et al. Determinants of plasma concentrations of perfluoroalkyl and polyfluoroalkyl substances in pregnant women from a birth cohort in Shanghai, China. Environ Int. 2018; 119:165-73.

4. Sagiv SK, Rifas-Shiman SL, Webster TF, Mora AM, Harris MH, Calafat AM, et al. Sociodemographic and perinatal predictors of early pregnancy perand Polyfluoroalkyl substance (PFAS) concentrations. Environ Sci Technol. 2015;49(19):11849-58.

5. Okada E, Kashino I, Matsuura H, Sasaki S, Miyashita C, Yamamoto J, et al. Temporal trends of perfluoroalkyl acids in plasma samples of pregnant women in Hokkaido, Japan, 2003-2011. Environ Int. 2013:60:89-96.

6. Cariou R, Veyrand B, Yamada A, Berrebi A, Zalko D, Durand S, et al. Perfluoroalkyl acid (PFAA) levels and profiles in breast milk, maternal and cord serum of French women and their newborns. Environ Int. 2015;84:71-81.

7. Bjerregaard-Olesen C, Bossi R, Liew Z, Long M, Bech BH, Olsen J, et al. Maternal serum concentrations of perfluoroalkyl acids in five international birth cohorts. Int J Hyg Environ Health. 2017;220(2 Pt A):86-93.

8. Wang T, Wang P, Meng J, Liu S, Lu Y, Khim JS, et al. A review of sources, multimedia distribution and health risks of perfluoroalkyl acids (PFAAs) in China. Chemosphere. 2015;129:87-99.

9. Land M, de Wit CA, Cousins IT, Herzke D, Johansson J, Martin JW. What is the effect of phasing out long-chain per- and polyfluoroalkyl substances on the concentrations of perfluoroalkyl acids and their precursors in the environment? A systematic review protocol. Environmental Evidence. 2015; 4(1):3.

10. Olsen G, Burris J, Ehresman D, Froehlich J, Seacat A, Butenhoff J, et al. Halflife of serum elimination of perfluorooctanesulfonate,perfluorohexanesulfonate, and perfluorooctanoate in retired fluorochemical production workers. Environ Health Perspect. 2007; 115(9):1298-305.

11. Yang L, Li J, Lai J, Luan H, Cai Z, Wang Y, et al. Placental transfer of Perfluoroalkyl substances and associations with thyroid hormones: Beijing prenatal exposure study. Sci Rep. 2016;6:21699.

12. Kim S, Choi K, Ji K, Seo J, Kho Y, Park J, et al. Trans-placental transfer of thirteen perfluorinated compounds and relations with fetal thyroid hormones. Environ Sci Technol. 2011;45(17):7465-72.

13. Lau C, Thibodeaux JR, Hanson RG, Rogers JM, Grey BE, Stanton ME, et al. Exposure to perfluorooctane sulfonate during pregnancy in rat and mouse. II: postnatal evaluation. Toxicol Sci. 2003;74(2):382-92.

14. Luebker DJ, York RG, Hansen KJ, Moore JA, Butenhoff JL. Neonatal mortality from in utero exposure to perfluorooctanesulfonate (PFOS) in SpragueDawley rats: dose-response, and biochemical and pharamacokinetic parameters. Toxicology. 2005;215(1):149-69.

15. Thibodeaux JR, Hanson RG, Rogers JM, Grey BE, Barbee BD, Richards JH, et al. Exposure to perfluorooctane sulfonate during pregnancy in rat and mouse. I: maternal and prenatal evaluations. Toxicol Sci. 2003;74(2):369-81.

16. Yu WG, Liu W, Jin YH, Liu XH, Wang FQ, Liu L, et al. Prenatal and postnatal impact of perfluorooctane sulfonate (PFOS) on rat development: a crossfoster study on chemical burden and thyroid hormone system. Environ Sci Technol. 2009;43(21):8416-22

17. Drover SSM, Villanger GD, Aase H, Skogheim TS, Longnecker MP, Zoeller RT, et al. Maternal thyroid function during pregnancy or neonatal thyroid function and attention deficit hyperactivity disorder: a systematic review. Epidemiology. 2019;30(1):130-44.

18. Lyall $K$, Anderson M, Kharrazi M, Windham GC. Neonatal thyroid hormone levels in association with autism spectrum disorder. Autism Res. 2017;10(4): 585-92.

19. Preston EV, Webster TF, Oken E, Claus Henn B, McClean MD, Rifas-Shiman SL, et al. Maternal plasma per- and Polyfluoroalkyl substance concentrations in early pregnancy and maternal and neonatal thyroid function in a prospective birth cohort: project viva (USA). Environ Health Perspect. 2018; 126(2):027013.

20. Wang Y, Rogan WJ, Chen PC, Lien GW, Chen HY, Tseng YC, et al. Association between maternal serum perfluoroalkyl substances during pregnancy and maternal and cord thyroid hormones: Taiwan maternal and infant cohort study. Environ Health Perspect. 2014;122(5):529-34.

21. Aimuzi R, Luo K, Chen Q, Wang H, Feng L, Ouyang F, et al. Perfluoroalkyl and polyfluoroalkyl substances and fetal thyroid hormone levels in umbilical cord blood among newborns by prelabor caesarean delivery. Environ Int. 2019;130:104929.

22. Shah-Kulkarni S, Kim BM, Hong YC, Kim HS, Kwon EJ, Park H, et al. Prenatal exposure to perfluorinated compounds affects thyroid hormone levels in newborn girls. Environ Int. 2016;94:607-13.

23. de Cock M, de Boer MR, Lamoree M, Legler J, van de Bor M. Prenatal exposure to endocrine disrupting chemicals in relation to thyroid hormone levels in infants - a Dutch prospective cohort study. Environ Health. 2014;13: 106.

24. Itoh S, Araki A, Miyashita C, Yamazaki K, Goudarzi H, Minatoya M, et al. Association between perfluoroalkyl substance exposure and thyroid hormone/thyroid antibody levels in maternal and cord blood: the Hokkaido study. Environ Int. 2019:133(Pt A):105139.

25. Tian Y, Liang H, Miao M, Yang F, Ji H, Cao W, et al. Maternal plasma concentrations of perfluoroalkyl and polyfluoroalkyl substances during pregnancy and anogenital distance in male infants. Hum Reprod. 2019;34(7): 1356-68.

26. Ji H, Liang H, Wang Z, Miao M, Wang X, Zhang X, et al. Associations of prenatal exposures to low levels of Polybrominated Diphenyl ether (PBDE) with thyroid hormones in cord plasma and neurobehavioral development in children at 2 and 4years. Environ Int. 2019;131:105010.

27. Bobb JF, Claus Henn B, Valeri L, Coull BA. Statistical software for analyzing the health effects of multiple concurrent exposures via Bayesian kernel machine regression. Environ Health. 2018;17(1):67.

28. Bobb JF, Valeri L, Claus Henn B, Christiani DC, Wright RO, Mazumdar M, et al. Bayesian kernel machine regression for estimating the health effects of multi-pollutant mixtures. Biostatistics. 2015;16(3):493-508.

29. Fan P, Luo Z, Tang N, Wang W, Liu Z, Zhang J, et al. Advanced maternal age, mode of delivery, and thyroid hormone levels in Chinese newborns. Front Endocrinol. 2019;10:913.

30. Li F, Yang F, Li DK, Tian Y, Miao M, Zhang Y, et al. Prenatal bisphenol a exposure, fetal thyroid hormones and neurobehavioral development in children at 2 and 4 years: a prospective cohort study. Sci Total Environ. 2020;722:137887.

31. Shi X, Liu C, Wu G, Zhou B. Waterborne exposure to PFOS causes disruption of the hypothalamus-pituitary-thyroid axis in zebrafish larvae. Chemosphere. 2009;77(7):1010-8.

32. Liu $Y$, Wang J, Fang $X$, Zhang $H$, Dai J. The thyroid-disrupting effects of long-term perfluorononanoate exposure on zebrafish (Danio rerio). Ecotoxicology. 2011;20(1):47-55.

33. Preston E, Webster T, Claus Henn B, McClean M, Gennings C, Oken E, et al. Prenatal exposure to per- and polyfluoroalkyl substances and maternal and neonatal thyroid function in the project viva cohort: a mixtures approach. Environ Int. 2020;139:105728.

34. Lebeaux R, Doherty B, Gallagher L, Zoeller R, Hoofnagle A, Calafat A, et al. Maternal serum perfluoroalkyl substance mixtures and thyroid hormone concentrations in maternal and cord sera: the HOME study. Environ Res. 2020;185:109395.

35. Tsai MS, Lin CC, Chen MH, Hsieh WS, Chen PC. Perfluoroalkyl substances and thyroid hormones in cord blood. Environ Pollut. 2017;222:543-8.

36. Dufour P, Pirard C, Seghaye MC, Charlier C. Association between organohalogenated pollutants in cord blood and thyroid function in newborns and mothers from Belgian population. Environ Pollut. 2018;238: 389-96.

37. Weiss JM, Andersson PL, Lamoree MH, Leonards PE, van Leeuwen SP, Hamers T. Competitive binding of poly- and perfluorinated compounds to the thyroid hormone transport protein transthyretin. Toxicol Sci. 2009;109(2): 206-16.

38. Yu WG, Liu W, Jin YH. Effects of perfluorooctane sulfonate on rat thyroid hormone biosynthesis and metabolism. Environ Toxicol Chem. 2009;28(5): 990-6.

39. Yu WG, Liu W, Liu L, Jin YH. Perfluorooctane sulfonate increased hepatic expression of OAPT2 and MRP2 in rats. Arch Toxicol. 2011;85(6):613-21. 
40. Tahboub R, Arafah BM. Sex steroids and the thyroid. Best Pract Res Clin Endocrinol Metab. 2009;23(6):769-80.

41. Kjeldsen LS, Bonefeld-Jorgensen EC. Perfluorinated compounds affect the function of sex hormone receptors. Environ Sci Pollut Res Int. 2013;20(11): 8031-44.

42. Biegel LB, Liu RC, Hurtt ME, Cook JC. Effects of ammonium perfluorooctanoate on Leydig cell function: in vitro, in vivo, and ex vivo studies. Toxicol Appl Pharmacol. 1995;134(1):18-25.

43. Itoh S, Araki A, Mitsui T, Miyashita C, Goudarzi H, Sasaki S, et al. Association of perfluoroalkyl substances exposure in utero with reproductive hormone levels in cord blood in the Hokkaido study on environment and Children's health. Environ Int. 2016;94:51-9.

\section{Publisher's Note}

Springer Nature remains neutral with regard to jurisdictional claims in published maps and institutional affiliations.

Ready to submit your research? Choose BMC and benefit from:

- fast, convenient online submission

- thorough peer review by experienced researchers in your field

- rapid publication on acceptance

- support for research data, including large and complex data types

- gold Open Access which fosters wider collaboration and increased citations

- maximum visibility for your research: over $100 \mathrm{M}$ website views per year

At BMC, research is always in progress.

Learn more biomedcentral.com/submissions 